\title{
Arachnoid calcification producing spinal cord compression
}

\author{
GLENN A. J. MCCULLOCH ${ }^{1}$ \\ From the Department of Surgery, \\ University of Tasmania, Hobart, Australia
}

SYNOPSIS A case of spinal cord compression, presumed to be due to a calcification in the arachnoid, is presented. Its relationship to a previous spinal subarachnoid haemorrhage is mentioned. The literature is reviewed and the relationship of this condition to spinal subarachnoid haemorrhage, previous myelogram, and spinal anaesthetic is stressed.

The spinal cord is a structure which is peculiarly prone to marked loss of function when compressed. The numerous conditions which may result in pressure upon the spinal cord and consequent loss of function include intervertebral discs, osteophytic bars, tumours, haematomas, and, of course, trauma. Arachnoid calcification is a very rare and disputed cause of spinal cord compression, but the case reported here is convincing. The relationship of this condition to subarachnoid haemorrhage is also noted.

\section{CASE HISTORY}

FIRST ADMISSION In May, 1970, this woman, who was then aged 62 years, presented to another hospital with a six day history of severe pain in the thoracic region of her spine, associated with vomiting, headache, and confusion. The pain radiated into the right flank and right thigh and was initially diagnosed as being due to renal colic. She had no previous history of any serious illnesses or any similar trouble. Examination revealed a marked degree of tenderness in the thoracic region, marked neck stiffness, positive straight leg raising test at $30^{\circ}$ bilaterally. There was decreased sensation to pinprick in her right leg and she was confused.

A lumbar puncture yielded evenly blood-stained spinal fluid, which produced no growth on culture. Serum calcium, urea, and electrolytes and blood haemoglobin were normal. Her ESR was $7 \mathrm{~mm}$ in the first hour. Radiograph of her lumbar spine, skull,

1 Address for reprints and correspondence: G. A. J. McCulloch, c/o Department of Neurosurgery, The Queen Elizabeth Hospital, Woodville Road, Woodville, S. Australia 5011.

(Accepted 25 June 1975.) and chest was within normal limits. Both vertebral and both carotid arteries were examined by arch aortography. The intracranial circulations of these vessels were all completely normal, and there was no evidence of aneurysm or an arteriovenous malformation. The patient required catheterization because of urinary retention. After six weeks' hospitalization,

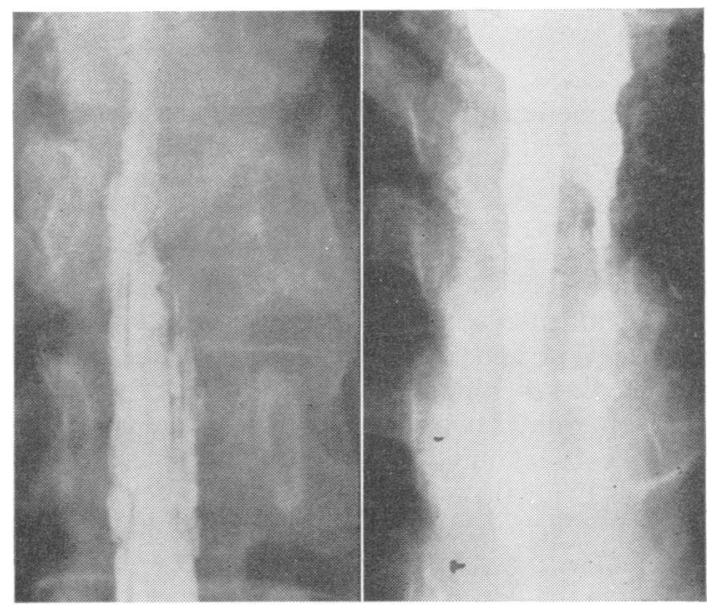

(1)

(2)

FIG. 1 Myelogram done before the first operation showing the complete block of the dye column at the 12 th thoracic vertebra. The dye column is also irregular due to the indentation with the arachnoid plaques.

FIG. 2 A cisternal myelogram done at the same time as Fig. 1 showing a large plaque from the fifth to seventh thoracic vertebra, with a complete block at the 1059 


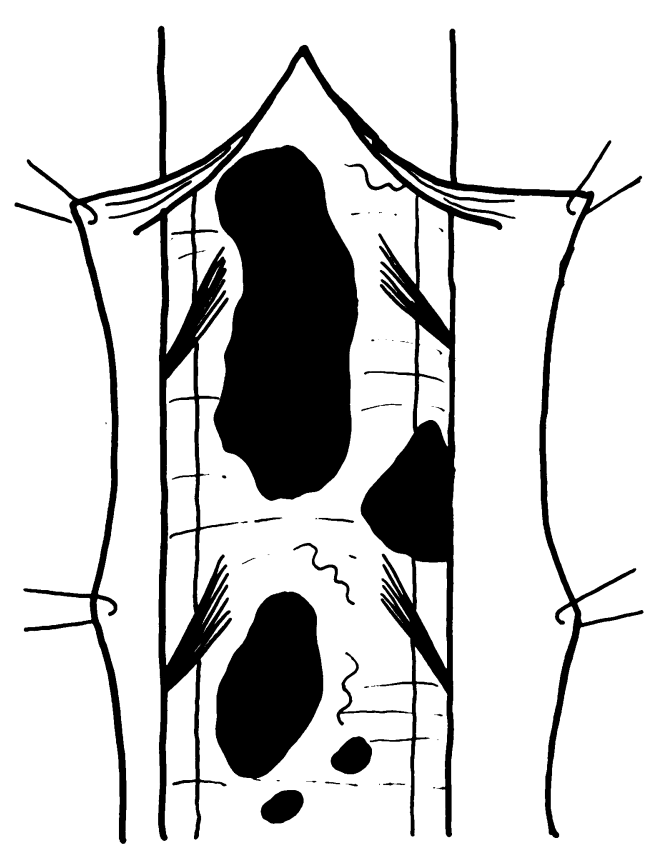

FIG. 3 A sketch of the findings at the first operation. The area depicted is the upper part of the laminectomy wound before the opening of the arachnoid. The areas of dense calcification are shown.

she was well enough to be able to walk around the ward unaided and was free of symptoms. She was discharged from hospital.

SECOND ADMISSION The patient remained well until approximately November 1972, when she started having difficulty with walking. This became gradually more severe and in September 1973, she was readmitted after falling over at home. By this time the patient required two walking sticks in order to walk.

Clinical examination revealed a spastic paraparesis. Cranial nerve function and the motor and sensory function of the arms were within normal limits. There was marked weakness of both legs, involving the flexor more than the extensor muscles, and involving the right more than the left leg. Both plantar responses were extensor and there was clonus bilaterally at the ankles. Vibration sensation was absent up to and including the iliac crests bilaterally; it was present at the rib margins. There was decreased perception of light touch and pinprick up to T8 dermatome on the left and T10 dermatome on the right, with a slight degree of sacral sparing. She was tender over the upper thoracic spine.

Radiographs of the thoracic spine revealed mild degenerative changes, but no other abnormality. A chest radiograph was normal. A myelogram performed by the lumbar route showed a complete block at the level of the 12th thoracic vertebra (Fig. 1). Iophendylate (Myodil) was injected into the cisterna magna and there was similarly demonstrated a complete block at the level of the 7 th thoracic vertebra, with some irregularity in the dye column between the 5th and 7th thoracic vertebrae (Fig. 2). A serum B12 level and blood haemoglobin were within normal limits. Laminectomy from $\mathrm{T} 5$ to $\mathrm{L} 1$ vertebrae, revealed an extensive area of calcification lying immediately deep to the dura mater. The areas of calcification were interspersed with normal areas of arachnoid; in total, there were 10 large pieces of calcification in the arachnoid, varying in size from about $0.5 \mathrm{~cm}$ in diameter to one very large piece, $1 \mathrm{~cm}$ by $5 \mathrm{~cm}$, which surrounded about half of the

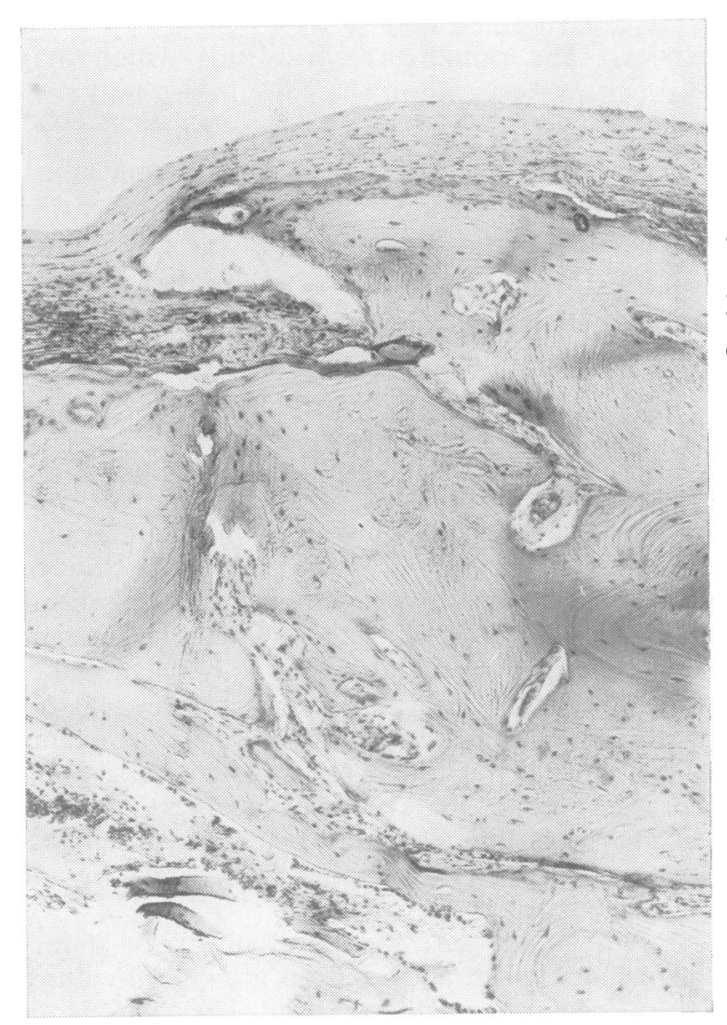

FIG. 4 A photomicrograph of one of the removed plaques. The outer surface (uppermost in picture) is smooth and lined with dura mater; the inner surface is irregular. The specimen is bone with well-developed Haversian systems. Haematoxylin and eosin, $\times 60$. 
circumference of the cord. This was located at the 7th thoracic vertebra (Fig. 3). At this level on the cord there was an indentation which exactly fitted the irregularities of the calcification. The thickest part of this area of calcification was $5 \mathrm{~mm}$ in depth. The outer surface of the calcification was smooth and immediately adjacent to the dura mater, whereas the inner surface was irregular and pitted. In several places, dorsal nerve roots ran through grooves on the surface of the calcification, and at one level, at the 10 th thoracic vertebra, the nerve root was completely enveloped by the calcification. The cord appeared normal; there was certainly no evidence of an arteriovenous malformation presenting on the dorsal surface. Histological examination of the removed tissue showed it to consist of lamellar bone without any evidence of neoplasm (Fig. 4).

Post-operatively, the patient's neurological state improved such that, six months after the operation,

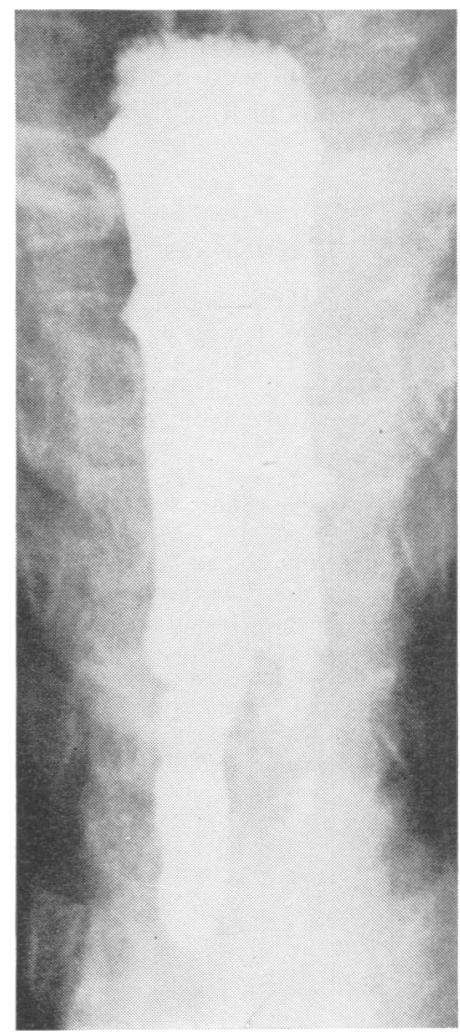

FIG. 5 A photograph of the myelogram (done by cisternal puncture) before the second operation. The area of complete obstruction is apparent with the single remaining plaque. she was able to walk around her home with minimal assistance and perform housework satisfactorily.

THIRD ADMISSION The patient presented again in March 1975, with a history that over the past four months she had had increasing difficulty in walking, with pain in her back going into her right thigh. She had also had some urinary incontinence. Clinical examination on this occasion revealed a remarkably similar picture to that present in October 1973, with a similar level of sensory loss and a similar pattern of motor loss in the lower extremities. Lumbar myelogram was attempted but no CSF could be obtained, even with the aid of an image-intensifier to locate correctly the lumbar puncture needle. A cisternal myelogram was therefore performed and showed a complete block at the level of the 5th thoracic vertebra (Fig. 5).

A second thoracic laminectomy on 24 February 1975 disclosed a single area of arachnoid calcification at the 5th dorsal vertebra, which was removed. Immediately postoperatively, there was some improvement in the sensation in the lower extremities, but three days postoperatively this worsened. Over the next four days there was marked fluctuation in the patient's motor and sensory function of the lower legs. She eventually settled to have a persistent loss of motor function in the lower extremities, with the exception of plantar flexion in the left foot. There was only mild perception of sensation in both lower extremities with the sensory level being at T7 dermatome bilaterally, without perineal sparing. Her condition remained virtually unchanged over the following two months.

\section{DISCUSSION}

Compression of the spinal cord with clinical symptoms due to calcified plaques in the arachnoid is very rare. Indeed, some authors have disputed whether this is ever the cause of clinical symptoms. Certainly, it is true that calcified and ossified plaques are a common postmortem finding in the arachnoid mater. Knoblich and Olsen (1966) examined 217 unselected necropsy patients. They found that $43.3 \%$ of the spinal cords and membranes examined had arachnoid calcification, most commonly in the lower thoracic and lumbar regions. Their description of some of the larger plaques corresponds well with the finding in this particular patient. They noted that the outer surface of the plaque is smooth and is immediately adjacent to the dura mater and that the inner surface is pitted and irregular. In 
some of their cases they found nerve roots passing through the area of calcification, as in our patient. Indeed, the illustration in their article showing extensive confluent plaques is identical with the appearance observed in our patient at the initial operation (Fig. 3). Furthermore, the histological findings of the plaques in their cases were similar to ours. We were unable to demonstrate the meningeal cells which they found on both the inner and outer layers of the plaques (Fig. 4). These authors conclude that these plaques are an extremely common finding, and that they often are falsely accused of being the cause of the patient's symptoms. Indeed, they state 'that, even in the symptomatic cases, the plaques are not the cause of the symptoms'. They do, however, qualify the statement by saying that it is possible that a single very large plaque may produce symptoms in a given patient.

So much then for the plaques which appear as a normal finding in so many patients. What then of the cases reported in which spinal cord compression has been attributed to these lesions? Various authors have attributed the cause of such symptom-producing plaques to four categories: (1) due to previous subarachnoid haemorrhage (Gatzke et al., 1957; Wise and Smith, 1965); (2) previous myelogram (Miles and Bhandari, 1971); (3) spinal anaesthetic (Wise and Smith, 1965); (4) previous trauma (Puusepp, 1931).

In the present case there was obviously a relationship between the previous subarachnoid haemorrhage and the arachnoid calcification. The subarachnoid haemorrhage appears to have been of spinal rather than intracranial origin, as evidenced by the cauda equina signs. This raises the possibility that the patient's spinal cord may, in fact, harbour an arteriovenous malformation (AVM) which is the cause of her symptoms rather than the arachnoid calcification. This certainly is a possibility, and we are unable to state categorically that the patient does not have an AVM. It was considered unwise for an elderly patient with impaired cord function to be submitted to a spinal angiogram, particularly since operative treatment would probably not have been possible as it was not a dorsally presenting arteriovenous malformation. However, the improvement after laminectomy and the marked indentation of the cord by the largest plaque makes one fairly certain that a significant part was played in the patient's cord symptoms by the calcification.

It is also interesting to note that, in keeping with other reports in the literature, the improvement after operation on this patient was not remarkable. Wise and Smith (1965) reported two cases with some improvement after the operation, but with later progressive deterioration of one of their patients. The patient reported by Nizzoli and Testa (1968) followed a similar course with increasing neurological loss three years after the operation.

\section{REFERENCES}

Gatzke, L. D., Dodge, H. W., and Dockerty, M. B. (1957). Arachnoiditis ossificans. Report of two cases. Proceedings of Mayo Clinic, 32, 689-704.

Knoblich, R., and Olsen, B. S. (1966). Calcified and ossified plaques of the spinal arachnoid membranes. Journal of Neurosurgery, 25, 275-279.

Miles J., and Bhandari, Y. S. (1971). Ossifying spinal arachnoiditis. Neurochirurgia (Stuttgart), 14, 184-188.

Nizzoli, V., and Testa, C. (1968). A case of calcification in the spinal arachnoid giving rise to spinal cord compression. Journal of the Neurological Sciences, 7, 381-384.

Puusepp, L. (1931). Surgical intervention in four cases of myelitis compression caused by osseous deposits in the arachnoidea of the spinal cord (arachnoiditis ossificans). Journal of Nervous and Mental Diseases, 73, 1-19.

Wise, B. L., and Smith, M. (1965). Spinal arachnoiditis ossificans. Archives of Neurology, 13, 391-394. 